\title{
On a Partition Function of Richard Stanley
}

\author{
George E. Andrews* \\ Department of Mathematics \\ The Pennsylvania State University \\ University Park, PA 16802 \\ andrews@math.psu . edu \\ Submitted: Sep 5, 2003; Accepted: Nov 19, 2003; Published: Jun 3, 2004 \\ MR Subject Classifications: 05A17 \\ In honor of my friend Richard Stanley
}

\begin{abstract}
In this paper, we examine partitions $\pi$ classified according to the number $r(\pi)$ of odd parts in $\pi$ and $s(\pi)$ the number of odd parts in $\pi^{\prime}$, the conjugate of $\pi$. The generating function for such partitions is obtained when the parts of $\pi$ are all $\leqq N$. From this a variety of corollaries follow including a Ramanujan type congruence for Stanley's partition function $t(n)$.
\end{abstract}

\section{Introduction}

Let $\pi$ denote a partition of some integer and $\pi^{\prime}$ its conjugate. For definitions of these concepts, see $[1 ;$ Ch.1]. Let $\mathcal{O}(\pi)$ denote the number of odd parts of $\pi$. For example, if $\pi$ is $6+5+4+2+2+1$, then the Ferrers graph of $\pi$ is

Reading columns we see that $\pi^{\prime}$ is $6+5+3+3+2+1$. Hence $\mathcal{O}(\pi)=2$ and $\mathcal{O}\left(\pi^{\prime}\right)=4$.

Richard Stanley ([4] and [5]) has shown that if $t(n)$ denotes the number of partitions $\pi$ of $n$ for which $\mathcal{O}(\pi) \equiv \mathcal{O}\left(\pi^{\prime}\right)(\bmod 4)$, then

\footnotetext{
*Partially supported by National Science Foundation Grant DMS-0200047
} 


$$
t(n)=\frac{1}{2}(p(n)+f(n))
$$

where $p(n)$ is the total number of partitions of $n[1, \mathrm{p} .1]$, and

$$
\sum_{n=0}^{\infty} f(n) q^{n}=\prod_{i \geqq 1} \frac{\left(1+q^{2 i-1}\right)}{\left(1-q^{4 i}\right)\left(1+q^{4 i-2}\right)^{2}} .
$$

Note that $t(n)$ is Stanley's partition function referred to in the title of this paper. Stanley's result for $t(n)$ is related nicely to a general study of sign-balanced, labeled posets [5]. In this paper, we shall restrict our attention to $S_{N}(n, r, s)$, the number of partition $\pi$ of $n$ where each part of $\pi$ is $\leqq N, \mathcal{O}(\pi)=r, \mathcal{O}\left(\pi^{\prime}\right)=s$. In Section 2, we shall prove our main result:

\section{Theorem 1.}

$$
\sum_{n, r, s \geqq 0} S_{2 N}(n, r, s) q^{n} z^{r} y^{s}=\frac{\sum_{j=0}^{N}\left[\begin{array}{c}
N \\
j
\end{array} ; q^{4}\right]\left(-z y q ; q^{4}\right)_{j}\left(-z y^{-1} q ; q^{4}\right)_{N-j}(y q)^{2 N-2 j}}{\left(q^{4} ; q^{4}\right)_{N}\left(z^{2} q^{2} ; q^{4}\right)_{N}},
$$

and

$$
\sum_{n, r, s \geqq 0} S_{2 N+1}(n, r, s) q^{n} z^{r} y^{s}=\frac{\sum_{j=0}^{N}\left[\begin{array}{c}
N \\
j
\end{array} ; q^{4}\right]\left(-z y q ; q^{4}\right)_{j+1}\left(-z y^{-1} q ; q^{4}\right)_{N-j}(y q)^{2 N-2 j}}{\left(q^{4} ; q^{4}\right)_{N}\left(z^{2} q^{2} ; q^{4}\right)_{N+1}},
$$

where

$$
\left[\begin{array}{c}
N \\
j
\end{array} ; q\right]= \begin{cases}\frac{\left(1-q^{N}\right)\left(1-q^{N-1}\right) \ldots\left(1-q^{N-j+1}\right)}{\left(1-q^{j}\right)\left(1-q^{j-1}\right) \ldots(1-q)}, & \text { for } 0 \leqq j \leqq N \\
0, & \text { if } j<0 \text { or } j>N\end{cases}
$$

and

$$
(A ; q)_{M}=(1-A)(1-A q) \ldots\left(1-A q^{M-1}\right) .
$$

From Theorem 1 follows an immediate lovely corollary:

\section{Corollary 1.1 .}

$$
\sum_{n, r, s \geqq 0} S_{\infty}(n, r, s) q^{n} z^{r} y^{s}=\prod_{j=1}^{\infty} \frac{\left(1+y z q^{2 j-1}\right)}{\left(1-q^{4 j}\right)\left(1-z^{2} q^{4 j-2}\right)\left(1-y^{2} q^{4 j-2}\right)} .
$$

From Corollary 1.1, we shall see in Section 3 that

\section{Corollary 1.2 .}

$$
t(5 n+4) \equiv 0 \quad(\bmod 5)
$$

Also, 


\section{Corollary 1.3.}

$$
\sum_{n=0}^{\infty} t(n) q^{n}=\frac{Q\left(q^{2}\right)^{2} Q\left(q^{16}\right)^{5}}{Q(q) Q\left(q^{4}\right)^{5} Q\left(q^{32}\right)^{2}}
$$

where

$$
Q(q)=(q ; q)_{\infty}=\prod_{j=1}^{\infty}\left(1-q^{j}\right)
$$

We conclude with some open questions.

\section{The Main Theorem}

We begin with some preliminaries about partitions and their conjugates. For a given partition $\pi$ with parts each $\leqq N$, we denote by $f_{i}(\pi)$ the number of appearances of $i$ as a part of $\pi$. The parts of $\pi^{\prime}$ in non-increasing order are thus

$$
\sum_{i=1}^{N} f_{i}(\pi), \sum_{i=2}^{N} f_{i}(\pi), \sum_{i=3}^{N} f_{i}(\pi), \ldots, \sum_{i=N}^{N} f_{i}(\pi) .
$$

Note that some of the entries in this sequence may well be zero; the non-zero entries make up the parts of $\pi^{\prime}$. However in light of the fact that 0 is even, we see that $\mathcal{O}\left(\pi^{\prime}\right)$ is the number of odd entries in the sequence (11) while

$$
\mathcal{O}(\pi)=f_{1}(\pi)+f_{3}(\pi)+f_{5}(\pi)+\ldots
$$

We now define

$$
\sigma_{N}(q, z, y)=\left(\sum_{n, r, s \geqq 0} S_{N}(n, r, s) q^{n} z^{r} y^{s}\right)\left(q^{4} ; q^{4}\right)_{\left\lfloor\frac{N}{2}\right\rfloor}\left(z^{2} q^{2} ; q^{4}\right)_{\left\lfloor\frac{N+1}{2}\right\rfloor} .
$$

Lemma 2.1. $\sigma_{0}(q, z, y)=1$, and for $N \geqq 1$,

$$
\begin{aligned}
\sigma_{2 N}(q, z, y) & =\sigma_{2 N-1}(q, z, y)+y^{2 N} q^{2 N} \sigma_{2 N-1}\left(q, z, y^{-1}\right) \\
\sigma_{2 N-1}(q, z, y) & =\sigma_{2 N-2}(q, z, y)+z y^{2 N-1} q^{2 N-1} \sigma_{2 N-2}\left(q, z, y^{-1}\right) .
\end{aligned}
$$

Proof. We shall in the following be dealing with partitions whose parts are all $\leqq$ some given $N$. We let $\bar{\pi}$ be that partition made up of the parts of $\pi$ that are $<N$. In light of (11) we see that if $N$ is a part of $\pi$ an even number of times, then $\mathcal{O}\left(\pi^{\prime}\right)=\mathcal{O}\left(\bar{\pi}^{\prime}\right)$ and if $N$ appears an odd number of times in $\pi$, then $\mathcal{O}\left(\bar{\pi}^{\prime}\right)=N-\mathcal{O}(\pi)$ (because the removal of $f_{N}(\pi)$ from each sum in (11) reverses parity). Initially we note that the only partition with at most zero parts is the empty partition of 0 ; hence $\sigma_{0}(q, z, y)=1$. 
Next, for $N \geqq 1$,

$$
\begin{aligned}
& \frac{\sigma_{2 N}(q, z, y)}{\left(q^{4} ; q^{4}\right)_{N}\left(z^{2} q^{2} ; q^{4}\right)_{N}} \\
= & \sum_{\pi, \operatorname{parts} \leqq 2 N} q^{\sum i f_{i}(\pi)} z^{f_{1}(\pi)+f_{3}(\pi)+\ldots+f_{2 N-1}(\pi)} y^{\mathcal{O}\left(\pi^{\prime}\right)} \\
= & \sum_{\substack{\pi, \operatorname{parts} \leq 2 N \\
f_{2 N}(\pi) \text { even }}} q^{\sum i f_{i}(\bar{\pi})+2 N f_{2 N}(\pi)} z^{f_{1}(\pi)+f_{3}(\pi)+\ldots+f_{2 N-1}(\pi)} y^{\mathcal{O}\left(\bar{\pi}^{\prime}\right)} \\
& +\sum_{\substack{\pi, \operatorname{parts} \leq 2 N \\
f_{2 N}(\pi) \text { odd }}} q^{\sum i f_{i}(\bar{\pi})+2 N f_{2 N}(\pi)} z^{f_{1}(\pi)+f_{3}(\pi)+\ldots+f_{2 N-1}(\pi)} y^{2 N-\mathcal{O}\left(\pi^{\prime}\right)} \\
= & \frac{1}{\left(1-q^{4 N}\right)} \frac{\sigma_{2 N-1}(q, z, y)}{\left(q^{4} ; q^{4}\right)_{N-1}\left(z^{2} q^{2} ; q^{4}\right)_{N}}+\frac{y^{2 N} q^{2 N}}{\left(1-q^{4 N}\right)} \frac{\sigma_{2 N-1}\left(q, z, y^{-1}\right)}{\left(q^{4} ; q^{4}\right)_{N-1}\left(z^{2} q^{2} ; q^{4}\right)_{N}},
\end{aligned}
$$

which is equivalent to (14).

Finally,

$$
\begin{aligned}
& \frac{\sigma_{2 N+1}(q, z, y)}{\left(q^{4} ; q^{4}\right)_{N}\left(z^{2} q^{2} ; q^{4}\right)_{N+1}} q^{\sum_{\pi, \operatorname{parts} \leq 2 N+1}^{\sum i f_{i}(\pi)} z^{f_{1}(\pi)+f_{3}(\pi)+\ldots+f_{2 N+1}(\pi)} y^{\mathcal{O}\left(\pi^{\prime}\right)}} \\
= & \sum_{\substack{\pi, \operatorname{parts} \leq 2 N+1 \\
f_{2 N+1}(\pi) \text { even }}} q^{\sum i f_{i}(\bar{\pi})+(2 N+1) f_{2 N+1}(\pi)} z^{f_{1}(\pi)+\ldots+f_{2 N+1}(\pi)} y^{\mathcal{O}\left(\bar{\pi}^{\prime}\right)} \\
& \left.+\sum_{\substack{\pi, \operatorname{parts} \leq 2 N+1 \\
f_{2 N+1}(\pi) \text { odd }}} q^{\sum i f_{i}(\bar{\pi})+(2 N+1) f_{2 N+1}(\pi)} z^{f_{1}(\pi)+\ldots+f_{2 N-1}(\pi)+f_{2 N+1}(\pi)} y^{2 N+1-\mathcal{O}\left(\bar{\pi}^{\prime}\right)}\right) \\
= & \frac{1}{\left(1-z^{2} q^{4 N+2}\right)} \frac{\sigma_{2 N}(q, z, y)}{\left(q^{4} ; q^{4}\right)_{N}\left(z^{2} q^{2} ; q^{4}\right)_{N}}+\frac{y^{2 N+1} q^{2 N+1} z}{\left(1-z^{2} q^{4 N+2}\right)} \frac{\sigma_{2 N}(q, z, y)}{\left(q^{4} ; q^{4}\right)_{N}\left(z^{2} q^{2} ; q^{4}\right)_{N}},
\end{aligned}
$$

which is equivalent to (15) with $N$ replaced by $N+1$.

Proof of Theorem 1. We let $\tau_{2 N}(q, z, y)$ denote the numerator on the right-hand side of (3) and $\tau_{2 N+1}(q, z, y)$ denote the numerator on the right-hand side of (4). If we can show that $\tau_{N}(q, z, y)$ satisfies (14) and (15), then noting immediately that $\tau_{0}(q, z, y)=1$, we will have proved that $\sigma_{N}(q, z, y)=\tau_{N}(q, z, y)$ for each $N \geqq 0$ (by mathematical induction) and will then prove Theorem 1 once we recall (13). 
First,

$$
\begin{aligned}
& \tau_{2 N-1}(q, z, y)+y^{2 N} q^{2 N} \tau_{2 N-1}\left(q, z, y^{-1}\right) \\
& =\sum_{j \geqq 0}\left[\begin{array}{c}
N-1 \\
j
\end{array} ; q^{4}\right]\left(-z y q ; q^{4}\right)_{j+1}\left(-z y^{-1} q ; q^{4}\right)_{N-j-1}(y q)^{2(N-1-j)} \\
& +y^{2 N} q^{2 N} \sum_{j \geqq 0}\left[\begin{array}{c}
N-1 \\
j
\end{array} ; q^{4}\right]\left(-z y^{-1} q ; q^{4}\right)_{N-j}\left(-z y q ; q^{4}\right)_{j}\left(y^{-1} q\right)^{2 j} \\
& \text { (where } j \rightarrow N-1-j \text { in the second sum) } \\
& =\sum_{j \geqq 0}\left[\begin{array}{c}
N-1 \\
j-1
\end{array} ; q^{4}\right]\left(-z y q ; q^{4}\right)_{j}\left(-z y^{-1} q ; q^{4}\right)_{N-j}(y q)^{2(N-j)} \\
& +y^{2 N} q^{2 N} \sum_{j \geqq 0}\left[\begin{array}{c}
N-1 \\
j
\end{array} ; q^{4}\right]\left(-z y^{-1} q ; q^{4}\right)_{N-j}\left(-z y q ; q^{4}\right)_{j}\left(y^{-1} q\right)^{2 j} \\
& \text { (where } j \rightarrow j-1 \text { in the first sum) } \\
& =\sum_{j \geqq 0}\left(-z y q ; q^{4}\right)_{j}\left(-z y^{-1} q ; q^{4}\right)_{N-j}(y q)^{2(N-j)}\left\{\left[\begin{array}{c}
N-1 \\
j-1
\end{array} ; q^{4}\right]+q^{4 j}\left[\begin{array}{c}
N-1 \\
j
\end{array} ; q^{4}\right]\right\} \\
& =\sum_{j \geqq 0}\left(-z y q ; q^{4}\right)_{j}\left(-z y^{-1} q ; q^{4}\right)_{N-j}(y q)^{2(N-j)}\left[\begin{array}{c}
N \\
j
\end{array} ; q^{4}\right] \\
& \text { (by }[1, \text { p.35, eq.(3.3.4)]) } \\
& =\tau_{2 N}(q, z, y) \text {. }
\end{aligned}
$$

Finally,

$$
\begin{aligned}
& \tau_{2 N}(q, z, y)+z y^{2 N+1} q^{2 N+1} \tau\left(q, z, y^{-1}\right) \\
& =\sum_{j=0}\left[\begin{array}{c}
N \\
j
\end{array} q^{4}\right]\left(-z y q ; q^{4}\right)_{j}\left(-z y^{-1} q ; q^{4}\right)_{N-j}(y q)^{2 N-2 j} \\
& \quad+z q^{2 N+1} y^{2 N+1} \sum_{j=0}^{N}\left[\begin{array}{c}
N \\
j
\end{array} ; q^{4}\right]\left(-z y^{-1} q ; q^{4}\right)_{N-j}\left(-z y q ; q^{4}\right)_{j}\left(q y^{-1}\right)^{2 j} \\
& \quad \quad(\text { where } j \rightarrow N-j \text { in the second sum }) \\
& =\sum_{j=0}^{N}\left[\begin{array}{c}
N \\
j
\end{array} ; q^{4}\right]\left(-z y q ; q^{4}\right)_{j}\left(-z y^{-1} q ; q^{4}\right)_{N-j}(y q)^{2 N-2 j}\left(1+z y q^{4 j+1}\right) \\
& =\sum_{j=0}^{N}\left[\begin{array}{c}
N \\
j
\end{array} ; q^{4}\right]\left(-z y q ; q^{4}\right)_{j+1}\left(-z y^{-1} q ; q^{4}\right)_{N-j}(y q)^{2 N-2 j} \\
& =\tau_{2 N+1}(q, z, y) .
\end{aligned}
$$


Proof of Corollary 1.1. From Theorem 1 (either (3) or (4) with $j \rightarrow N-j$ ),

$$
\begin{aligned}
& \sum_{n, r, s \geqq 0} S_{\infty}(n, r, s) q^{n} z^{r} y^{s} \\
& =\frac{1}{\left(q^{4} ; q^{4}\right)_{\infty}\left(z^{2} q^{2} ; q^{4}\right)_{\infty}} \sum_{j=0}^{\infty} \frac{1}{\left(q^{4} ; q^{4}\right)_{j}}\left(-z y q ; q^{4}\right)_{\infty}\left(-z y^{-1} q ; q^{4}\right)_{j}(y q)^{2 j} \\
& =\frac{\left(-z y q ; q^{4}\right)_{\infty}}{\left(q^{4} ; q^{4}\right)_{\infty}\left(z^{2} q^{2} ; q^{4}\right)_{\infty}} \frac{\left(-z y q^{3} ; q^{4}\right)_{\infty}}{\left(y^{2} q^{2} ; q^{4}\right)_{\infty}} \\
& =\frac{(\text { by }[1, \mathrm{p} \cdot 17, \text { eq. }(2.2 .1)])}{\left(q^{4} ; q^{4}\right)_{\infty}\left(z^{2} q^{2} ; q^{4}\right)_{\infty}\left(y^{2} q^{2} ; q^{4}\right)_{\infty}}
\end{aligned}
$$

which is Corollary 1.1.

Corollary 2.1. Identity (1) is valid.

Proof. We note that $\mathcal{O}(\pi) \equiv \mathcal{O}\left(\pi^{\prime}\right)(\bmod 2)$ because each is clearly congruent $(\bmod 2)$ to the number being partitioned. Hence,

$$
\begin{aligned}
\sum_{n \geqq 0} t(n) q^{n} & =\sum_{\substack{n, r, s \geqq 0 \\
\frac{r-s}{2} \text { even }}} S_{\infty}(n, r, s) q^{n} \\
& =\frac{1}{2} \sum_{n, r, s \geqq 0} S_{\infty}(n, r, s) q^{n}\left(1+i^{r-s}\right) \\
& =\frac{1}{2}\left(\frac{\left(-q ; q^{2}\right)_{\infty}}{\left(q^{4} ; q^{4}\right)_{\infty}\left(q^{2} ; q^{4}\right)_{\infty}^{2}}+\frac{\left(-q ; q^{2}\right)_{\infty}}{\left(q^{4} ; q^{4}\right)_{\infty}\left(-q^{2} ; q^{4}\right)_{\infty}^{2}}\right) \\
& =\frac{1}{2}\left(\frac{1}{(q ; q)_{\infty}}+\frac{\left(-q ; q^{2}\right)_{\infty}}{\left(q^{4} ; q^{4}\right)_{\infty}\left(-q^{2} ; q^{4}\right)_{\infty}^{2}}\right) \\
& =\frac{1}{2} \sum_{n=0}^{\infty}(p(n)+f(n)) q^{n},
\end{aligned}
$$

and comparing coefficients of $q^{n}$ in the extremes of this identity we deduce (1).

\section{$3 \quad$ Further Properties of $t(n)$}

Corollary 1.2. $t(5 n+4) \equiv 0(\bmod 5)$.

Proof. Ramanujan proved [3, p.287, Th. 359] that

$$
p(5 n+4) \equiv 0 \quad(\bmod 5) .
$$

So it follows from (1) that to prove $5 \mid t(5 n+4)$ we need only prove that $5 \mid f(5 n+4)$. 
By (2),

$$
\begin{aligned}
\sum_{n=0}^{\infty} f(n) q^{n} & =\frac{\left(-q ; q^{2}\right)}{\left(q^{4} ; q^{4}\right)_{\infty}\left(-q^{2} ; q^{4}\right)_{\infty}^{2}} \\
& =\frac{\left(-q ; q^{4}\right)_{\infty}\left(-q^{3} ; q^{4}\right)_{\infty}\left(q^{4} ; q^{4}\right)_{\infty}}{\left(q^{4} ; q^{4}\right)_{\infty}^{2}\left(-q^{2} ; q^{4}\right)_{\infty}^{2}} \\
& =\frac{1}{\left(-q^{2} ;-q^{2}\right)_{\infty}^{2}} \sum_{n=-\infty}^{\infty} q^{2 n^{2}-n} \quad(\text { by }[1, \text { p.21, eq. }(2.2 .10)]) \\
& =\frac{\left(-q^{2} ;-q^{2}\right)_{\infty}^{3}}{\left(-q^{2} ;-q^{2}\right)_{\infty}^{5}} \sum_{n=-\infty}^{\infty} q^{2 n^{2}-n} \\
& =\frac{1}{\left(-q^{10} ;-q^{10}\right)_{\infty}} \sum_{n=\infty}^{\infty} q^{2 n^{2}-n} \sum_{j=0}^{\infty}(-1)^{j+(j+1) / 2}(2 j+1) q^{j^{2}+j}
\end{aligned}
$$

(by [3, p.285, Thm. 357]).

Now the only time an exponent of $q$ in the numerator is congruent to $4(\bmod 5)$ is when $n \equiv 4(\bmod 5)$ and $j \equiv 2(\bmod 5)$. But then $(2 j+1) \equiv 0(\bmod 5)$, i.e. the coefficient of $q^{5 m+4}$ in the numerator must be divisible by 5 . Given that the denominator is a function of $q^{5}$, it cannot possibly affect the residue class of any term when it is divided into the numerator. So,

$$
f(5 n+4) \equiv 0 \quad(\bmod 5)
$$

Therefore,

$$
t(5 n+4) \equiv 0 \quad(\bmod 5)
$$

\section{Corollary 1.3.}

$$
\sum_{n \geqq 0} t(n) q^{n}=\frac{Q(q)^{2} Q\left(q^{16}\right)^{5}}{Q(q) Q\left(q^{4}\right)^{5} Q\left(q^{32}\right)^{2}}
$$

where

$$
Q(q)=(q ; q)_{\infty}
$$


Proof. By (17),

$$
\begin{aligned}
\sum_{n \geqq 0} t(n) q^{n} & =\frac{1}{2}\left(\frac{\left(-q ; q^{2}\right)_{\infty}}{\left(q^{4} ; q^{4}\right)_{\infty}\left(q^{2} ; q^{4}\right)_{\infty}^{2}}+\frac{\left(-q ; q^{2}\right)_{\infty}}{\left(q^{4} ; q^{4}\right)_{\infty}\left(-q^{2} ; q^{4}\right)_{\infty}^{2}}\right) \\
& =\frac{\left(-q ; q^{2}\right)_{\infty}}{2\left(q^{4} ; q^{4}\right)_{\infty}^{2}\left(q^{2} ; q^{4}\right)_{\infty}^{2}\left(-q^{2} ; q^{4}\right)_{\infty}^{2}}\left(\left(q^{4} ; q^{4}\right)_{\infty}\left(-q^{2} ; q^{4}\right)_{\infty}^{2}+\left(q^{4} ; q^{4}\right)_{\infty}\left(q^{2} ; q^{4}\right)_{\infty}^{2}\right) \\
& =\frac{\left(-q ; q^{2}\right)_{\infty}}{2\left(q^{4} ; q^{4}\right)_{\infty}^{2}\left(q^{4} ; q^{8}\right)_{\infty}^{2}}\left(\sum_{n=-\infty}^{\infty} q^{2 n^{2}}+\sum_{n=-\infty}^{\infty}(-1)^{n} q^{2 n^{2}}\right) \\
& =\frac{\left(-q ; q^{2}\right)_{\infty}}{\left(q^{4} ; q^{4}\right)_{\infty}^{2}\left(q^{4} ; q^{8}\right)_{\infty}^{2}} \sum_{n=-\infty}^{\infty} q^{8 n^{2}} \\
& =\frac{\left(-q ; q^{2}\right)_{\infty}\left(q^{16} ; q^{16}\right)_{\infty}\left(-q^{8} ; q^{16}\right)_{\infty}^{2}}{\left(q^{4} ; q^{4}\right)_{\infty}^{2}\left(q^{4} ; q^{8}\right)_{\infty}^{2}} \\
& =\frac{Q\left(q^{2}\right)^{2} Q\left(q^{16}\right)^{5}}{Q(q) Q\left(q^{4}\right)^{5} Q\left(q^{32}\right)^{2}},
\end{aligned}
$$

where the last line follows from several applications of the two identities

$$
\left(q ; q^{2}\right)_{\infty}=\frac{Q(q)}{Q\left(q^{2}\right)}
$$

and

$$
\left(-q ; q^{2}\right)_{\infty}=\frac{Q\left(q^{2}\right)^{2}}{Q(q) Q\left(q^{4}\right)}
$$

Corollary 1.3 allows us to multisect the generating function for $t(n)$ modulo 4 .

\section{Corollary 3.1.}

$$
\begin{aligned}
\sum_{n \geqq 0} t(4 n) q^{n} & =\left(q^{16} ; q^{16}\right)_{\infty}\left(-q^{7} ; q^{16}\right)_{\infty}\left(-q^{9} ; q^{16}\right)_{\infty} W(q), \\
\sum_{n \geqq 0} t(4 n+1) q^{n} & =\left(q^{16} ; q^{16}\right)_{\infty}\left(-q^{5} ; q^{16}\right)_{\infty}\left(-q^{11} ; q^{16}\right)_{\infty} W(q), \\
\sum_{n \geqq 0} t(4 n+2) q^{n} & =q\left(q^{16} ; q^{16}\right)_{\infty}\left(-q ; q^{16}\right)_{\infty}\left(-q^{15} ; q^{16}\right)_{\infty} W(q), \\
\sum_{n \geqq 0} t(4 n+3) q^{n} & =\left(q^{16} ; q^{16}\right)_{\infty}\left(-q^{3} ; q^{16}\right)_{\infty}\left(-q^{13} ; q^{16}\right)_{\infty} W(q),
\end{aligned}
$$

where

$$
W(q)=\frac{Q\left(q^{4}\right)^{5}}{Q(q)^{5} Q\left(q^{8}\right)^{2}}
$$


Proof. We begin with Gauss's special case of the Jacobi Triple Product Identity [1, p.23, eq. $(2.2 .13)]$

$$
\sum_{n=-\infty}^{\infty} q^{2 n^{2}-n}=\frac{\left(q^{2} ; q^{2}\right)_{\infty}}{\left(q ; q^{2}\right)_{\infty}}=\frac{Q\left(q^{2}\right)^{2}}{Q(q)}
$$

Therefore by Corollary 1.3, we see that

$$
\sum_{n \geqq 0} t(n) q^{n}=W\left(q^{4}\right) \sum_{n=-\infty}^{\infty} q^{2 n^{2}-n}
$$

Now $2 n^{2}-n \equiv n(\bmod 4)$. So to obtain (3.4)-(3.7) we multisect the right-hand series in (27) by setting $n=4 m+j(0 \leqq j \leqq 3)$, so

$$
\sum_{n \geqq 0} t(n) q^{n}=W\left(q^{4}\right) \sum_{j=0}^{3} \sum_{m=-\infty}^{\infty} q^{2(4 m+j)^{2}-(4 m+j)} .
$$

One then obtains four identities arising from the four residue classes mod 4 . We carry out the full calculations in the case $j=0$ :

$$
\begin{aligned}
\sum_{n \geqq 0} t(4 n) q^{4 n} & =W\left(q^{4}\right) \sum_{m=-\infty}^{\infty} q^{32 m^{2}-4 m} \\
& =W\left(q^{4}\right)\left(q^{64} ; q^{64}\right)_{\infty}\left(-q^{28} ; q^{64}\right)_{\infty}\left(-q^{36} ; q^{64}\right)_{\infty}
\end{aligned}
$$

a result equivalent to (3.4) once $q$ is replaced by $q^{1 / 4}$. The remaining results are proved similarly.

\section{Conclusion}

As is obvious, Theorem 1 is easily proved once it is stated, but the sums appearing in (3) and (4) seem to arise from nowhere.

I note that by considering the cases $N=1,2,3,4$, I discovered empirically that

$$
\sum_{n, r, s \geqq 0} S_{2 N}(n, r, s) q^{n} z^{r} y^{s}=\frac{1}{\left(q^{4} ; q^{4}\right)_{N}} \sum_{j=0}^{N} \frac{\left(-z y q ; q^{2}\right)_{2 j}}{\left(z^{2} q^{2} ; q^{4}\right)_{j}}\left[\begin{array}{c}
N \\
j
\end{array} ; q^{4}\right]\left(y^{2} q^{2}\right)^{N-j}
$$

and

$$
\sum_{n, r, s \geqq 0} S_{2 N+1}(n, r, s) q^{n} z^{r} y^{s}=\frac{1}{\left(q^{4} ; q^{4}\right)_{N}} \sum_{j=0}^{N} \frac{\left(-z y q ; q^{2}\right)_{2 j+1}}{\left(z^{2} q^{2} ; q^{4}\right)_{j+1}}\left[\begin{array}{c}
N \\
j
\end{array} ; q^{4}\right]\left(y^{2} q^{2}\right)^{N-j}
$$

One can then pass to (3) and (4) by means of a ${ }_{3} \phi_{2}$ transformation [2, p.242, eq.(III.13)], and the proof of Theorem 1 is easiest using (3) and (4). 
The referee notes that both (1.3) and (1.4) can be written as a ${ }_{2} \phi_{1}$. These ${ }_{2} \phi_{1}$ series can both be transformed into ${ }_{3} \phi_{1}$ series, equivalent to (1.4) and (4.2) by (III.8) of [2].

There are many mysteries surrounding many of the identities in this paper.

Problem 1. Is there a partition statistic that will divide the partitions enumerated by $t(5 n+4)$ into five equinumerous classes? Dyson's rank (largest part minus number of parts) provides such a division at least for $n=0$ and 1 (cf. [1, p.175]).

Problem 2. Identity (7) cries out for combinatorial proof.

I have been informed that A. Sills, A. J. Yee, and C. Boulet have independently found such proofs in addition to further results.

\section{References}

[1] G.E. Andrews, The Theory of Partitions, Addison-Wesley, Reading, 1976 (Reissued: Cambridge University Press, Cambridge, 1998).

[2] G. Gasper and M. Rahman, Basic Hypergeometric Series, Cambridge University Press, Cambridge, 1990.

[3] G.H. Hardy and E.M. Wright, An Introduction to the Theory of Numbers, 4 ed., Oxford University Press, Oxford, 1960.

[4] R.P. Stanley, Problem 10969, Amer. Math. Monthly, 109 (2002), 760.

[5] R.P. Stanley, Some remarks on sign-balanced and maj-balanced posets (to appear). 